\title{
SNORA42 Promote Oesophageal Squamous Cell Carcinoma Development Through Interacting With Dhx9
}

\section{Yanan Shan}

Hebei Medical University Fourth Affiliated Hospital and Hebei Provincial Tumor Hospital https://orcid.org/0000-0001-7844-3571

\section{Sisi Wei}

Hebei Medical University Fourth Affiliated Hospital and Hebei Provincial Tumor Hospital

\section{Xiaohao Xiang}

Hebei Medical University Fourth Affiliated Hospital and Hebei Provincial Tumor Hospital

\section{Suli Dai}

Hebei Medical University Fourth Affiliated Hospital and Hebei Provincial Tumor Hospital

\section{Wenxuan Cui}

Hebei Medical University Fourth Affiliated Hospital and Hebei Provincial Tumor Hospital

\section{Riyang Zhao}

Hebei Medical University Fourth Affiliated Hospital and Hebei Provincial Tumor Hospital

\section{Cong Zhang}

Hebei Medical University Fourth Affiliated Hospital and Hebei Provincial Tumor Hospital

\section{Weimin Zhang}

Chinese Academy of Medical Sciences and Peking Union Medical College

Lianmei Zhao ( $\sim$ zhaolianmei@hbydsy.com )

https://orcid.org/0000-0002-5854-9827

\section{Baoen Shan}

Hebei Medical University Fourth Affiliated Hospital and Hebei Provincial Tumor Hospital

\section{Research}

Keywords: SNORA42, Oesophageal Squamous Cell Carcinoma, DHX9, p65

Posted Date: May 29th, 2020

DOI: https://doi.org/10.21203/rs.3.rs-31392/v1

License: (c) (1) This work is licensed under a Creative Commons Attribution 4.0 International License. 


\section{Abstract}

Background:Oesophageal squamous cell carcinoma (ESCC) is a high malignant cancer, which is the most common subtype of oesophageal cancer. Small nucleolar RNAs (snoRNAs) are a group of novel non-coding RNAs that have been found play a key role in various cancers.

Methods:The expression of SNORA42 in ESCC samples and cell lines was measured by using real-time PCR and a series of in vitro and in vivo assays were performed to determine the function of SNORA42 in ESCC. Furthermore, RNA pull-down assay combined with Mass Spectrometry was applied to identify the protein that is associated with SNORA42. Silencing SNORA42 in ESCC cell followed by Next-Generation mRNA Sequencing was used to investigate the signaling pathway regulated by SNORA42.

Results: We identified H/ACA box snoRNA42 (SNORA42) wasupregulated in ESCC and had the potential to be applied as a prognostic marker. Specifically, overexpression of SNORA42

promotedESCCprogression in vitro and in vivo, whereas knockdown of SNORA42 had opposite effects. We identified SNORA42 interacted with DHX9, which was up-regulated in ESCC and had a positive correlation with the expression of SNORA42. Furthermore, the promotion of SNORA42 on ESCC phenotypes can be reversed by knockdown of DHX9. Mechanically, SNORA42 promoted DHX9 expression by attenuating DHX9 transports into cytoplasm, to protect DHX9 from being ubiquitinated and degraded. From KEGG analysis of Next-Generation Sequencing, the NF-KB pathway was one of the most regulated pathways by SNORA42. SNORA42 enhanced phosphorylation of p65 and this effect could be reversed by NF-KB inhibitor, BAY11-7082. Moreover, SNORA42 activated NF-KB signaling through promotingthe transcriptional co-activator DHX9 interacted with p-p65, inducingNF-KB downstream genes expression.

Conclusions:These findings suggest that SNORA42 is up-regulated in ESCC and promotes ESCC developments,partly via interacting with DHX9 and triggering SNORA42/DHX9/p65 axis.

\section{Introduction}

Oesophageal cancer is one of the most human malignant cancers with the 7th incidence rate and the 6th mortality rate over the world[1]. Oesophageal squamous cell carcinomas (ESCC) is the most common histologic subtype of oesophageal cancer[2]-[4]. However, there is no specific symptom for ESCC in early stages; thus, in most cases, it cannot be detected until advanced stages accompany organ metastasis leading to miss best therapy opportunity. Identification of early diagnosis biomarkers of ESCC is an ideal screening strategy while there are fewer studies about it[5].

Small nucleolar RNAs (snoRNAs) is a group of conserved noncoding RNAs with 60-300nt in length[6]. It has been classified into 2 groups: C/D box snoRNAs (SNORDs) and H/ACA box snoRNAs (SNORAs). C/D box snoRNAs contain a closed loop which is consisted with $\mathrm{C}$ and $\mathrm{D}$ motif whereas $\mathrm{H} / \mathrm{ACA}$ box snoRNAs contain 2 loops linked by $\mathrm{H}$ box and ACA motif located at 3' end[7]. snoRNAs have been well known to exert its function through binding to ribonucleoprotein (RNP) to form snoRNP[8]-[10]. H/ACA box 
small nuclear RNAs (snRNAs)[7], [11]. However, recent studies have revealed novel roles of snoRNAs in human disease[12], included cancer[13], [14]. Moreover, more evidence indicated that snoRNAs may be treated as potential biomarkers in clinical application[15], [16].

SNORA42 is an H/ACA box snoRNA and encoded by KIAA0907. The previous study indicated that SNORA42 was overexpressed in non-small cell lung cancer (NSCLC) and promoted tumorigenicity in a p53-dependent manner[17]. In addition, overexpressed SNORA42 was positively correlated with poor prognosis in NSCLC[15],[17]. Another study revealed that SNORA42 expression was negative related with overall survival, disease-free survival and a risk factor for distant metastasis in colorectal cancer. In this study, SNORA42 was also identified the patients with high risk for recurrence and poor prognosis in stage II colorectal cancer[16]. Most recently, SNORA42 has been reported to enhance prostate cancer tumorgenesis by promoting EMT progression[18]. However, there is no research about the role of SNORA42 in ESCC.

DEAH box protein 9 (DHX9), also named ATP dependent RNA helicase A (RHA) is a member of the DEAH family of human RNA helicases[19]. DHX9 has been reported upregulated in different human cancers and promoted tumor process through interacting with a number of transcription factors [20]- [25]. The previous study reported that DHX9 interacted with nuclear factor-KB (NF-KB) dimer p65 and enhanced NF$\kappa B$ transcriptional ability, leading to NF-KB downstream genes expression[22].

The aim of this study was to investigate the function of SNORA42 in ESCC. Here, we found that SNORA42 is upregulated in ESCC patient's tissues and serum, and has a positive relationship with poor survival and metastasis. Furthermore, SNORA42 promoted ESCC development ability by interacting with DHX9 to enhance ESCC proliferation, migration and invasion ability. On one hand, SNORA42 blocks ubiquitination of DHX9 to prolong DHX9 lifespan. On the other hand, SNORA42 enhances the interaction between DHX9 and p-p65 to trigger NF-KB activity, and then promotes transcription of NF-KB target genes.

\section{Materials And Methods}

\section{Cell Culture and transient transfection}

All ESCC cell lines (Eca-109, KYSE30, TE-1, KYSE510, KYSE180, KYSE170, TE-13, and YES2) and immortalized human oesophageal epithelial cells (NE2-hTERT) were obtained from Cell Culture Center, Chinese Academy of Medical Sciences (Beijing, China). ESCC cell lines were cultured with RPMI 1640 (Invitrogen) media with 10\% fetal calf serum (Hyclone, Logan, UT), as well as penicillin and streptomycin (Invitrogen) at $37^{\circ} \mathrm{C}$ with $5 \% \mathrm{CO}_{2}$. NE2-hTERT cell line was cultured with 1:1 ratio of defined keratinocyteSFM (DKSFM) and epilife medium with EDGS. Cells were transiently transfected with a plasmid (Invitrogen), siRNAs (sequences are listed in supplementary table 1) or ASO (RiboBio) using Lipofectamine 2000 or Lipofectamine ${ }^{\circledR}$ LTX with PlusTM Reagent (Life Technologies, Gaithersburg, MD). 
Two sets of samples were used in this study. The first set, which contained ESCC tissues and paired paracancer tissues, was collected from the Fourth Hospital of Hebei Medical University during 2014-2015. No patients received chemotherapy or radiation therapy before surgery. Informed consent for the use of samples was obtained from patients and approval was obtained from the hospital. All samples information was shown in Table 1. The other set, which contained serum from ESCC patients before surgery and control serum from healthy volunteers with age in 25-55. Informed consent for the use of samples was obtained from patients and volunteer, and approval was obtained from the hospital. 
Table 1

The relationship between SNORA42 expression and clinical pathological characteristics

\begin{tabular}{|c|c|c|c|c|}
\hline \multirow[t]{2}{*}{ Parameters } & \multirow[t]{2}{*}{ Total } & \multicolumn{2}{|c|}{ Expression of SNORA42 } & \multirow[t]{2}{*}{$P$-value } \\
\hline & & Low & High & \\
\hline \multicolumn{4}{|l|}{ Age/year } & \multirow[t]{3}{*}{0.418} \\
\hline$<60$ & 42 & 19 & 23 & \\
\hline$\geq 60$ & 58 & 31 & 27 & \\
\hline \multicolumn{4}{|l|}{ Gender } & \multirow[t]{3}{*}{0.235} \\
\hline Male & 77 & 36 & 41 & \\
\hline Female & 23 & 14 & 9 & \\
\hline \multicolumn{4}{|l|}{ Differentiation } & \multirow[t]{3}{*}{0.216} \\
\hline Well & 38 & 16 & 22 & \\
\hline Poor & 62 & 34 & 28 & \\
\hline \multicolumn{5}{|l|}{ TNM } \\
\hline \multicolumn{4}{|l|}{$\mathrm{T}$} & \multirow[t]{4}{*}{$0.019^{a}$} \\
\hline T1 & 14 & 11 & 3 & \\
\hline T2 & 18 & 13 & 5 & \\
\hline T3 & 46 & 17 & 29 & \\
\hline \multicolumn{4}{|l|}{$\mathbf{N}$} & \multirow[t]{3}{*}{$0.001^{a}$} \\
\hline NO & 58 & 36 & 22 & \\
\hline N1 & 42 & 14 & 28 & \\
\hline \multicolumn{4}{|l|}{ M } & \multirow[t]{3}{*}{$0.012^{a}$} \\
\hline MO & 87 & 48 & 39 & \\
\hline M1 & 13 & 2 & 11 & \\
\hline \multicolumn{4}{|l|}{ Stage } & \multirow[t]{3}{*}{$0.004^{a}$} \\
\hline ૧ & 9 & 5 & 4 & \\
\hline ૧ & 45 & 32 & 13 & \\
\hline \multicolumn{5}{|c|}{$\begin{array}{l}\text { Abbreviations: T, the size of original tumour; N, the nearby lymph nodes being invaded; } M \text {, the distant } \\
\text { metastasis. For analysis of correlation between SNORA42 and clinical features, the Chi-Squared Test } \\
\text { was utilized. The median value of expression level was used as the separated point. }{ }^{\text {Tathe values had }} \\
\text { Loading [MathJax]/jax/output/CommonHTML/jax.js }\end{array}$} \\
\hline
\end{tabular}




\begin{tabular}{|c|c|c|c|c|}
\hline \multirow[t]{2}{*}{ Parameters } & \multirow[t]{2}{*}{ Total } & \multicolumn{2}{|c|}{ Expression of SNORA42 } & \multirow[t]{2}{*}{$P$-value } \\
\hline & & Low & High & \\
\hline प & 34 & 9 & 25 & \\
\hline प & 12 & 4 & 8 & \\
\hline \multicolumn{4}{|l|}{ Lymphatic invasion } & \multirow[t]{3}{*}{$0.006^{a}$} \\
\hline Negative & 51 & 32 & 19 & \\
\hline Positive & 49 & 18 & 31 & \\
\hline \multicolumn{5}{|c|}{$\begin{array}{l}\text { Abbreviations: T, the size of original tumour; } \mathrm{N} \text {, the nearby lymph nodes being invaded; } \mathrm{M} \text {, the distant } \\
\text { metastasis. For analysis of correlation between SNORA } 42 \text { and clinical features, the Chi-Squared Test } \\
\text { was utilized. The median value of expression level was used as the separated point. }{ }^{\mathrm{a}} \text { The values had } \\
\text { statistically significant differences. }\end{array}$} \\
\hline
\end{tabular}

\section{Rna Extraction, Reverse Transcription, And Real-time Pcr}

Total RNA was extracted from cells, tissues or serum using TRIzol reagent (Invitrogen). The mRNA reverse transcription was synthesized by using MMLV reverse transcriptase (Promega) according to the manufacturer's instructions. Real-time PCR was performed using the QuantiTect SYBR Green PCR Kit (Takara Bio Inc., Otsu, Japan). Primer sequences were listed in supplementary table 1. The gene level was normalized to GAPDH or U6. Relative expression levels were calculated using the $2^{-\Delta \Delta C t}$ method. Each experiment was performed in at least 3 biological repetitions.

\section{Fluorescence In Situ Hybridization (fish) Assay}

The FISH assay was performed on Eca-109 cell to determine the cellular location of SNORA42. Cells were seeded on slides and fixed with $4 \%$ paraformaldehyde for $20 \mathrm{~min}$ at room temperature. Quantum dotfluorescent in situ hybridization (QD-FISH) was performed to detect the presence of SNORA42 using a digoxin-labeled oligonucleotide probe (RiboBio) indirectly labeled with streptavidin-conjugated quantum dots. 4', 6-diamidino-2-phenylindole (DAPI) was used as a marker for nucleus and 18S rRNA was used to indicate cytoplasm.

\section{Immunofluorescence Assay}

Immunofluorescence was performed on Eca-109 and KYSE30 cells to determine the subcellular localization of DHX9. The cell was seeded and fixed on a slice, followed blocked by $2 \%$ BSA and incubated with DHX9 antibody overnight. In the second day, slides were incubated with fluorescencelabeled (IF488, Abcam) secondary antibody, and DAPI to mark the location of the nucleus. The slide was Loading [MathJax]/jax/output/CommonHTML/jax.js :ope (LSM 700). 


\section{Proliferation And Colony Assays}

Proliferation and colony assays were performed to determine cell proliferation ability. Briefly, transfected cell was seeded in 96-well plate (1000/well), treated with Cell Titer 96 Aqueous on radioactive cellproliferation-assay(MTS,3-(4,5-dimethylthiazol-2-yl)-5-(3carboxymethoxyphenyl)-2-(4-sulfophenyl)-2Htetazolium)) and incubated for $2 \mathrm{~h}$ at $37^{\circ} \mathrm{C}$, followed by measured under $492 \mathrm{~nm}$ with a microplate reader.

\section{Wound Healing, Migration And Invasion Assays}

Wound healing, migration and invasion assays were used to investigate cell metastasis ability in vitro. Briefly, for wound healing assay, cells were seeded in 6-well plate and transfected. After $24 \mathrm{~h}$, scratched on confluence cell with a pipette tip, changed the media to serum-free media and graphed from 10X microscope. For migration and invasion assays, cells were seeded $(100,000 /$ well) in the upper chamber (24-well insert; Corning Costar, Cambridge, MA, USA) with (invasion) or without (migration) $200 \mu \mathrm{L} / \mathrm{ml}$ Matrigel (Beyotime Biotechnology) and after $20 \mathrm{~h}$, took photos from a microscope. Migrated and invaded cell numbers were automatically counted using a plugin of ImageJ.

\section{Animal Experiments (xenograft Assay And Vein Tail Transfer Assay)}

All male BALB/c nude mice (4-6 weeks old) were obtained from the Vital River Laboratory Animal Technology Co. Ltd., Beijing. Animal experiments contain two parts. The first one, nude mice (4- 6 weeks old) were injected subcutaneously under left armpit with SNORA42 knockdown cells and control cells (1 $\times 10^{6} /$ mouse, $n=6$ /group). Each group contains 6 nude mice. Tumor size was measured every 5 days until its volume up to $1500 \mathrm{~mm}^{3}$. 27 days later, the tumor was removed to weigh and used to be performed IHC assay. The other part, nude mice were separated into 2 groups $(n=6)$ randomly and

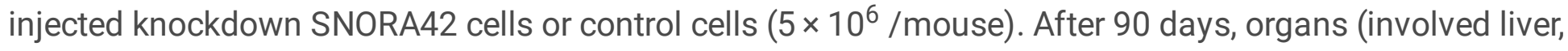
lung, brain) were removed and tested through H\&E staining assay to determine if organ metastasis occurred.

\section{Immunohistochemistry Staining Assay (ihc)}

Immunohistochemistry staining was performed on $4 \mu \mathrm{m}$-thick slices following EnVision two-step procedure of Dako REAL ${ }^{\text {TM }}$ Envision ${ }^{\text {TM }}$ Detection System (Dako). Different antibodies were used to incubate slices overnight, included Ki-67(1:100, Abcam), DHX9 (1:200, Proteintech), MMP2 (1:100, Abcam), MMP9 (1:500, Abcam). In the second day, slices were incubated with HRP labeled secondary antibody and visualized by diaminobenzidine. Score for percentage: <5\% (0), 5\%-25\% (1), 25\%-50\% (2), 50\%-75\% (3), 
and > 75\% (4); for intensity: no staining (0), light brown (1), brown (2), and dark brown (3). Each slice was assessed by two pathologists independently.

\section{H\&e Staining}

H\&E staining was used to determine the situation of tissue suspected cancer. Slices were dewaxed and dehydrated independently, followed by being stained with hematoxylin for 5-15 min and chromatic separated with alcohol hydrochloride. The nucleus was alkalized with lithium carbonate until the color of it became blue. Then slides were stained with eosin for 1-5 min, followed by dehydrated and covered with neutral balsam. Each slide was assessed by two pathologists independently.

\section{Protein Extraction And Immunoblotting}

Cell lysates and immunoblotting were prepared as described previously[26].Protein was detected with antibodies against DHX9 (1:1000, 17721-1-AP, Proteintech), MMP2 (1:500, ab97779, abcam), MMP9 (1:500, ab38898, abcam), p-p65(1:500, 3033, CST), Ubiquitin (1:1000, 3936, CST), smad2 (1:1000, 5399, CST), Bcl-2 (1:1000, 15071, CST), Bax (1:1000, 5023, CST), GAPDH (1:10000, 600-401-GV0S, ROCKLAND).

\section{Biotin-rna Pull-down Assay}

The pGEM-3zf(+)-SNORA42 and pGEM-3zf(+)-SNORA42-antisense plasmid were linearized to be used as templates for the transcription of SNORA42 and anti-SNORA42, followed by amplified through RT-PCR. Biotin-labeled RNAs were transcribed using Biotin-RNA Labeling Mix (Roche, Indianapolis, IN, USA), treated with RNase-free DNase I (TaKaRa, Kyoto, Japan) and purified with an RNeasy Mini Kit (Roche). Biotinylated RNAs were heated at $98^{\circ} \mathrm{C}$ for $2 \mathrm{~min}$, put on ice for $5 \mathrm{~min}$ and left at RM $25 \mathrm{~min}$ to form secondary structure. Total protein lysates were mixed with the Biotinylated RNA incubated at $30{ }^{\circ} \mathrm{C}$ and added Streptavidin agrose beads (GE Healthcare, Little Chalfont, UK) incubated at RM with rotation. The RNA-protein-beads complex washed with binding buffer, included RNA nuclease and proteinase, followed by boiled with loading buffer for 10 min. RNA affinity was tested by $12 \%$ sodium dodecyl sulfatepolyacrylamide gel electrophoresis and visualized by silver staining. The specific bands were excised and identified by mass spectrometry.

\section{Rip Assay}

RIP assay was used to confirm the interaction between protein and RNA. The assay was performed according to the manufacturer protocol of Magna RIP kit (Millipore, USA). The antibody against DHX9 was used to form the DHX9-SNORA42-magnetic beads complex, followed detected by RT-PCR and agrose 
gel. Total RNA was used as input and anti-rabbit IgG antibody was used as negative control to determine the RNA was specific binding to protein DHX9.

\section{Co-immunoprecipitation (co-ip) Assay}

Co-immunoprecipitation was performed to evaluate the effect of SNORA42 on the interaction between DHX9 and p-p65, as well as DHX9 and ubiquitin. Briefly, cell lysate was incubated with DHX9 overnight in the same quantification for each group. In the second day, the cell lysate and antibody complex was added magnetic beads to obtain a protein that specifically interacted with DHX9, followed boiled for 10 min and performed immunoblotting with antibodies against p-p65 (1:500, CST)and Ubiquitin (1:1000, CST). Reverse verification was performed for further confirmation. The total cell lysate was used as input and anti-rabbit IgG antibody was used as negative control.

\section{Statistical analysis}

All in vitro experiments were done at least 3 biological replicates in each group with repeated at least 3 times. The results are evaluated as mean \pm SEM in bar graphs and line graphs. Statistical analyses such as, paired or unpaired student's t-test, $\chi^{2}$ test or Mann-Whitney $U$ test, Pearson Correlation test were used through SPSS version 13.0 and GraphPad Prism 8.

\section{Results}

\section{Clinical significance of SNORA42 in ESCC}

Using ESCC tissue samples from The Fourth Hospital of Hebei Medical University, we found that SNORA42 expression in ESCC tissues was significantly higher than that in para-cancer tissues (Fig. 1a). From the analysis of TCGA database, SNORA42 level in oesophageal carcinoma was also significantly higher than that in normal oesophageal tissues (Fig. 1b) [27]. To further confirm the impact of SNORA42 in ESCC development, the analysis about SNORA42 and clinic pathological features of ESCC was performed. Patients with ESCC were divided into low-expression group and high-expression group based on median value of SNORA42 expression level in all patients. As shown in Table 1, high SNORA42 level was associated with tumor invasion, region lymph nodes invasion, distant metastasis and oesophageal cancer stage. We also tested the level of SNORA42 in patient's peripheral serum with early stage ESCC. To note, the serum was measured before patients got surgery, chemotherapy and radiotherapy. As shown in Fig. 1c, SNORA42 was significantly upregulated in ESCC patient's serum than in healthy serum. Moreover, SNORA42 expression in ESCC cell lines was higher than oesophageal immortalized epithelial cell $\left(\mathrm{NE}_{2}{ }^{-}\right.$ hTERT), especially, in Eca-109, KYSE30, and KYSE180 (Fig. 1d). So we will use these cell lines to investigate the function of SNORA42 in our following experiment.In addition, Kaplan-Meier survival analysis determined that patients with higher SNORA42 level were negatively correlated with 5-year 


\section{SNORA42 exerts tumor-promoting effects in vitro and in vivo}

The up-regulation of SNORA42 in ESCC tissue, serum and cell lines has been determined, however the role of SNORA42 in ESCC is still unknown. The expression of SNORA42 was significantly increased in KYSE30, Eca-109, and TE-1 cells after transfected with SNORA42 plasmid (Additional Fig. 1a, see Additional file 2). Due to the nuclear location and structure of SNORA42 constrained the knockdown efficiency of RNA interference (Additional Fig. 1b, see Additional file 2), antisense oligonucleotides (ASOs) was used to suppress endogenous SNORA42 level. The level of SNORA42 was significantly suppressed in KYSE30 and Eca-109 cells by ASOs (Additional Fig. 1c, see Additional file 2). Cell proliferation, migration and invasion were tested by MTS assay, Transwell migration assay, Transwell matrigel assay and wound-healing assay respectively. Overexpressing SNORA42 promoted cell proliferation, migration and invasion (Fig. 2a, b, Additional Fig. 1c, d, see Additional file 2) whereas knockdown of SNORA42 significantly inhibited these phenotypes (Fig. 2c, d, Additional Fig. 1f, see Additional file 2).

To further investigate the function of SNORA42 on ESCC in vivo, SNORA42 stably down-regulated cell line was generated using Lentivirus infected KYSE30 (Additional Fig. 1g, see Additional file 2). Both subcutaneous xenograft and tail vein injection nude mice models were utilized with the SNORA42 knockdown cell. In the subcutaneous xenograft mice model, the size and weight of tumor from SNORA42downregulated group were significantly inhibited compared with the control group (Fig. 2e, f). Moreover, the expression of cell proliferation marker Ki-67, metastasis markers MMP2 and MMP9 in tumor tissues was significantly suppressed (Additional Fig. $1 \mathrm{~h}$, see Additional file 2). In the tail vein injection metastasis model, nude mice in SNORA42-downregulated group had less distant organ metastasis confirmed by H\&E staining assay with 1 nude mouse had lung metastasis, 1 nude mouse had brain metastasis and none of them had liver metastasis. However, nude mice in the control group all had lung metastasis, 5 nude mice had brain metastasis and no liver metastasis (Fig. 2g, h). In summary, SNORA42 promoted ESCC proliferation, migration and invasion in vivo and in vitro.

\section{Snora42 Interacts With Dhx9 Protein}

To investigate the mechanism that how SNORA42 performs its promotion function in ESCC, a biotinlabeled RNA pull-down assay combined with mass spectrometric analysis was performed to identify the nucleus proteins that might interact with SNORA42. Three bands were found specifically interacted with biotinylated SNORA42 oligo (Fig. 3a). The liquid chromatography-mass spectrometric analysis was heterogeneous nuclear ribonucleoprotein and showed that 75 proteins potentially bound to SNORA42 (Additional Table 2, see Additional file 1). Among the obtained proteins, DHX9 is one of the highest abundance proteins that interacted with SNORA42. DHX9 is a cancer-related protein in DEAH family of human RNA helicases. It has been shown dysregulated in various human cancers and promotes tumor developments[20]- [25]. To validate whether DHX9 was associated with SNORA42, a series of 
biotinylated SNORA42 oligos whereas it cannot be pulled down by antisense of SNORA42 or nonbiotinylated SNORA42 oligos. Furthermore, the result of RNA immunoprecipitation (RIP) assay showed that SNORA42 was specifically enriched by anti-DHX9 antibody in ESCC cells (Fig. 3c). As shown in Fig. 3d and e, the cellular location of SNORA42 was in the nucleus which was confirmed by Fluorescence in situ hybridization (FISH) assay, and it was the same location with DHX9. These results indicated that SNORA42 was associated with DHX9 in ESCC cells.

\section{Snora42 Promotes Escc Progress Through Interacting With Dhx9}

Though DHX9 has been reported as an oncogene in multiple cancers, there is no report in ESCC. We tested the level of $D H X 9$ gene in ESCC clinical tissues and found that $D H X 9$ level was upregulated in ESCC (Fig. 3f). Knockdown of DHX9 significantly suppressed ESCC cells proliferation, migration, and invasion (Additional Fig. 2b, c, see Additional file 2). To test if SNORA42 exerts its function through associating with DHX9 in ESCC, We first tested whether the expression of DHX9 is associated with SNORA42 level in ESCC cells. The result showed that SNORA42 can suppress DHX9 protein expression in ESCC cells but had no effects on $D H X 9$ gene level (Fig. $3 \mathrm{~g}$ and Additional Fig. 2a, see Additional file 2). This result was coincident with ESCC clinical tissues, SNORA42 expression had a positive relationship with DHX9 protein in the same cohort clinical tissues in immunohistochemistry (IHC) assay, and DHX9 was more accumulated in nucleus in ESCC compared with para-cancer which was consistent with FISH assay (Fig. 3i). Furthermore, the IHC assay about mice subcutaneous xenograft tumour showed that DHX9 expression was significantly suppressed after knocking down SNORA42 (Fig. 3h). These results suggested that SNORA42 is associated with DHX9 in ESCC and has a positive correlation with DHX9 protein expression.

Whether the association of SNORA42 and DHX9 has an effect in ESCC development was evaluated by co-transfecting SNORA42 and siDHX9 in ESCC cells. As shown in Fig. 4a, the enhanced proliferation by SNORA42 was reversed by knocking down of DHX9. Moreover, the promotion of migration and invasion were partly rescued by knocking down of DHX9 (Fig. 4b and Additional Fig. 2d, see Additional file 2). Taken together, these results indicated that SNORA42 exerts its promotion function partly through interacting with DHX9 in ESCC.

\section{Snora42 Attenuates Dhx9 Ubiquitination}

Above studies verified that SNORA42 enhanced DHX9 protein level, but little is known about molecular mechanism. Given the results that SNORA42 played no effects on DHX9 gene level (Additional Fig. 2a, see Additional file 2), we hypothesized that SNORA42 might regulate DHX9 protein level in a posttranslation modification way, such as ubiquitination. Firstly, we determined whether SNORA42 affects half-life of DHX9 protein. Cvcloheximide (CHX, a protein biosynthesis inhibitor) was used to block DHX9 Loading [MathJax]/jax/output/CommonHTML/jax.js 
protein generation, and half-life period of DHX9 protein was measured. Specifically, ESCC cells were transfected with SNORA42 plasmid or vector, and treated with $\mathrm{CHX}$. Cell lysate was collected at different times after treatment. As shown in Fig. 4c, DHX9 protein level decreased 50\% with $\mathrm{CHX}$ treatment 4 hours in the control group, whereas in SNORA42 overexpression group half-life of DHX9 was dramatically delayed until later than 6 hours. Furthermore, we used MG132, a proteasome inhibitor, to protect DHX9 protein from being degraded. From Fig. 4d, knockdown of SNORA42 reduced DHX9 protein level whereas treated with MG132 blocked this effect. These results suggested that SNORA42 promoted DHX9 expression in ESCC cells by attenuated its protein degradation.

Ubiquitin-proteasome pathway is known involved in protein degradation of eukaryotic cells. The degradation process of DHX9 was still unknown so we firstly used immunoprecipitation assays with antiDHX9 antibody in ESCC cells to determine if DHX9 degradation through the ubiquitin-proteasome pathway. As shown in Fig. 4e, the ubiquitinated DHX9 was detected by the anti-ubiquitin antibody. Next, we tested our hypothesis that SNORA42 might involve in DHX9 ubiquitin degradation pathway. ESCC cells were transfected with SNORA42 plasmid followed by MG132 treatment, and Immunoprecipitation assay was performed. As shown in Fig. 4f, overexpression of SNORA42 reduced the DHX9 ubiquitination level, indicating that SNORA42 promoted DHX9 protein expression by attenuating DHX9 ubiquitination.

To further investigate how SNORA42 promoted DHX9 protein level, we measured the DHX9 in ESCC cell nucleus and cytoplasm. As shown in Additional Fig. 2e (see Additional file 2), SNORA42 could protect DHX9 accumulated in the nucleus from being translated into cytoplasm. Taken together, SNORA42 associated with DHX9 to accumulate DHX9 in nucleus, resulting to protect DHX9 protein from being degraded.

\section{Snora42 Regulates Nf-kb Pathway In Escc Cells}

To determine the molecular mechanism of SNORA42 regulating ESCC development, we performed the Next Generation Sequencing on KYSE30 after knocking down SNORA42 (GSE146195) and the sequencing result was verified by real-time PCR (Fig. 5a). It was found that in the genes that changed greater than 5 times between control and SNORA42 low-expressed groups, 70 genes were down-regulated whereas 8 genes were up-regulated (Additional Table 3, see Additional file 1). From KEGG analysis of the sequencing, we found that NF-KB was one of most regulated and cancer-related pathways by SNORA42 (Additional Fig. 3a, see Additional file 2). To investigate if SNORA42 could regulate the NF-kB pathway, we measured downstream genes of NF-KB after overexpressing and silencing SNORA42. It was shown that MMP2, MMP9, VEGFC, and BCL2 levels were changed consistent with SNORA42 expression (Fig. 5b). In addition, we tested two metastasis markers MMP2 and MMP9, which are also NF-KB downstream genes. As shown in Fig. $5 \mathrm{c}$ and d, protein and secreted levels of MMP2 and MMP9 were significantly increased after overexpressing SNORA42 whereas silencing SNORA42 reduced secreted MMP2 and MMP9. Next, we found that p-p65 level was enhanced by overexpression of SNORA42 and the 
inhibitor of NF-KB pathway, BAY11-7082 could reverse this promoted effect (Fig. 5e). These results suggested that SNORA42 activated NF-KB pathway in ESCC cells.

\section{SNORA42 promotes DHX9 interacted with p-p65 to regulate the NF-kB pathway}

Studies have revealed that DHX9 interacts with p65 and plays as an transcriptional co-activator to promote p65 downstream gene transcription [28], [29], we hypothesized that SNORA42 might regulate the interaction of p65 and DHX9 to modulate NF-KB signalling. We performed Co-Immunoprecipitation assay to test whether SNORA42 affected the association of p-p65 and DHX9. It was found that overexpression of SNORA42 increased the interaction of p-p65 and DHX9 in both Eca-109 and KYSE30 cells (Fig. 6a). In addition, SNORA42-induced NF-KB pathway downstream genes (MMP2, MMP9, VEGFC, and BCL2) by was reversed by knocking down of DHX9 (Fig. 6b). These results indicated that SNORA42 promoted pp65 and DHX9 interaction to enhance p65 transcription ability.

As the schematic graph shown in Fig. 6d, SNORA42 is over-expressed in ESCC to promote migration, invasion and proliferation in vitro and in vivo, in part through associating with $\mathrm{DHX} 9$ and promoting its protein level, furthermore activating p-p65 to promote target gene expression and finally regulating the biological activity.

\section{Discussion}

snoRNA has been considered as the house-keeping gene for a long time to guide rRNA modifications. The snoRNAs are conserved non-coding RNAs existing in different species and encoded from the introns of genomic genes. However, recent evidence reported their abnormal expressions in various human cancers, indicated that these snoRNAs might have other functions in human disease. It has been shown that snoRNAs, especially C/D box snoRNAs, are highly expressed in human acute myelogenous leukemia (AML)[30]. Studies also showed that both two classes of snoRNAs were significantly overexpressed in human colorectal cancer (CSC)[31]. Therefore, we selected several snoRNAs that overexpressed in ESCC from TCGA database (data not shown)[27]. We measured these snoRNAs expression in human ESCC tissues and found SNORA42 was one of the most highly expressed snoRNA. Furthermore, studies revealed that snoRNAs were as variable as miRNA expression and can be used potential biomarkers for early detection and prognostication of non-small cell lung cancers (NSCLC)[32]. Note, SNORA42 expression has been found negatively correlated with overall survival in an additional independent cohort and identified the patients with high risk for recurrence and poor prognosis in stage II CRC[31]. In this study, it analyzed the correlation between SNORA42 expression and clinical pathology characteristics to test the possibility of SNORA42 as a prognostic biomarker in ESCC.

The significant high expression of SNORA42 in ESCC and the positive relation with the patient's clinical characteristics are hoth sumanesting that the change of SNORA42 might have important roles in cell Loading [MathJax]/jax/output/CommonHTML/jax.js 
behaviour. It has been reported SNORA42 promoted cell growth and colony formation partially in a p53dependent manner[33]. In addition, Studies showed that SNORA42 enhanced prostate cancer cell viability, migration and epithelial-to-mesenchymal transition (EMT)[34]. In this study, we used two ways to knockdown and overexpress SNORA42 respectively to test the function of SNORA42 in ESCC cells. Moreover, we utilized two in vivo models further confirmed SNORA42 as an oncogene in ESCC.

DExH-Box Helicase 9 (DHX9) is a member of the DEAH-containing family of RNA helicases which has the ability to bind RNAs and catalyze the ATP-dependent unwinding of double-stranded RNAs (dsRNAs)[35]. It has been reported it is dysregulated in multiple human cancers[20], [21]. In this study, we found that the overexpression of SNORA42 suppressed the degradation of DHX9 and ubiquitination of DHX9, whereas had no effects on DHX9 mRNA level, indicating that SNORA42 promotes DHX9 expression by inhibiting its degradation, not the generation. Consistently, the expressions of DHX9 and SNORA42 correlated closely with ESCC clinical tissues and in vivo. We thought the reason might be the interaction of SNORA42 and DHX9 in nucleus attenuates DHX9 being transported to cytoplasm and degradation while further studies still needed. The recent study showed that SNORA18L 5 reduces $p 53$ expression by retaining RPL5 and RPL11 in the nucleolus that less RPL5 and RPL11 binding with MDM2 and inhibits its ubiquitin ligase activity toward p53 resulting p53 accumulation in hepatocellular carcinoma[36]. It also might be other reasons outside of the nucleus, as the study reported that the intracellular location of snoRNA changed from the nucleolus and other parts of the cell[8].

p65 (also named RELA) plays a key role in canonical NF-KB pathway inducing target gene transcription. p65 translocates into the nucleus and being phosphorylated by various kinases to trigger its interaction with CBP/p300 and thereby increases p65 transcription activity[37]. Numerous studies reported that pp65 involved in different tumorgenesis processes by promoting growth, proliferation, angiogenesis, and invasion[38]. Moreover, nuclear NF-kB p65 phosphorylation frequently existed in head and neck squamous cell cancer and NF-KB downstream genes are significantly upregulated[39].

A study revealed that the loss of amino-terminal enhancer of split (AES) interacts with multiple snoRNAs resulting in decreased leukemia self-renewal potential in AML[40]. Here, we demonstrated that SNORA42 promoted ESCC growth, migration and invasion partly through interacting with DHX9. It has been reported that DHX9 directly bind to nucleolar p65 and mediate the transcription activity of NF-KB[22]. Consistently, the KEGG analysis of next-generation sequencing showed the NF-KB pathway was one of the most modified pathways after overexpression of SNORA42. A study showed that the loss of SNORD50A and SNORD50B direct binding to K-Ras in human cancers leading to activate the K-Ras and synergize the effects of KRAS mutation[8]. Therefore, we hypothesized that SNORA42 might work as a scaffold to associate with both p65 and DHX9, but from RNA pull-down assay, neither p-p65 nor p65 associated with SNORA42. Then we hypothesis it might due to SNORA42 can regulate the interaction between DHX9 and p-p65. We found that overexpression of SNORA42 can promote their interaction and activate downstream genes of NF-kB expression, including metastasis-related genes in vitro and in vivo. 
The H/ACA box snoRNAs are well studied for post-transcriptional modification through rRNAs pseudouridylation. Studies reported that the DEAH-containing family has the ability to regulate rRNAs modification through interacting snoRNAs[40], [41]. So further studies are needed to determine the effects of interaction of DHX9 and SNORA42 on rRNA modifications, and whether they play roles through pseudouridylation or other pathways. Moreover, we found that SNORA42 enhanced DHX9 expression through inhibiting its degradation but not determine how SNORA42 prevents ubiquitination of DHX9.

The present study is the first to demonstrate that SNORA42 as an oncogene in ESCC through interacting with DHX9 and promoting DHX9 interact with p65 in the nucleus. The study has significant implications regarding the understanding of the roles of snoRNAs in ESCC pathogenesis, providing evidence for snoRNAs as biological prognosis markers in clinical ESCC treatment.

\section{Conclusions}

Taken together, we investigate an H/ACA box snoRNA, SNORA42 that is up-regulated in ESCC tissues and cells, and it is associated with poor prognosis. We found that SNORA42 promotes ESCC proliferation, metastasis in vitro and in vivo. Furthermore, SNORA42 associates with DHX9 to accumulate DHX9 in nucleus, leading to promote DHX9 protein level. The accumulated DHX9 further interacts with $\mathrm{p} 65$ to activate NF-KB signalling pathway, resulting up-regulation of the NF-KB downstream genes. Our study suggests that SNORA42 plays an oncogene in ESCC development, partly via interacting with DHX9 and triggering SNORA42/DHX9/p65 axis.

\section{Abbreviations}

snoRNA

Small nucleolar RNAs; ESCC:Oesophageal squamous cell carcinoma; SNORA42:H/ACA box snoRNA42; DHX9:DEAH box protein 9; NF-KB:Nuclear factor-kB; FISH:Fluorescence in situ hybridization; IHC:Immunohistochemistry; RIP:RNA immunoprecipitation; IF:Immunofluorescence; IP:Immunoprecipitation; ASO:Anti-sense oligonucleotide; TCGA:The Cancer Genome Atlas;

\section{Declarations}

\section{Ethics approval and consent to participate}

All the animal experiments were conducted according to the protocol approved by the Animal Care and Ethics Committee of The Fourth Hospital of Hebei Medical University. This study was carried out accordance with the Ethics Committee of The Fourth Hospital of Hebei Medical University.

\section{Consent for publication}




\section{Availability of data and materials}

The datasets and materials used in this study are available based on reasonable requested.

\section{Competing interests}

The authors have viewed the manuscript and declared that no conflict of interest exists.

\section{Funding}

This work was supported by the National Natural Science Foundation of China (Grant No. 81772550 , 81673642, 81973520), the Youth Outstanding Foundation of Hebei Province (Grant No. H2019206697), the Financial Department of Hebei Province (Grant No.20180498).

\section{Authors' contributions}

LZ, BS and YS designed and supervised this study; YS and SW performed the experiments; $X X$ helped with in vivo experiments and collecting clinical data; SD helped with analyzing data sets; WC helped with perform RNA Pull-down Assay and collecting clinical tissue samples; RZ and CZ helped with collecting clinical tissue samples and in vivo experiments; WZ helped with offering ESCC cell lines;

\section{Acknowledgements}

We are very grateful to all the patients and mice for their contributions and sacrifices to this study.

\section{Author details}

${ }^{1}$ Research Center, the Fourth Hospital of Hebei Medical University, Shijiazhuang, China;

2 State Key Laboratory of Molecular Oncology, Cancer institute and Cancer Hospital, Chinese Academy of Medical Sciences and Peking Union Medical College, Beijing 100021, China.

${ }^{3}$ Department of Biology, York University, Toronto, Canada

\section{References}


2. Lagergren J, Smyth E, Cunningham D, Lagergren P. Oesophageal cancer. The Lancet. Nov. 2017;390(10110):2383-96. doi:10.1016/S0140-6736(17)31462-9.

3. 10.1136/gutjnl-2014-308124

Arnold M, Soerjomataram I, Ferlay J, Forman D, "Global incidence of oesophageal cancer by histological subtype in 2012," Gut, vol. 64, no. 3, pp. 381-387, Mar. 2015, doi: 10.1136/gutjnl-2014308124.

4. Torre LA, Bray F, Siegel RL, Ferlay J, Lortet-Tieulent J, Jemal A. Global cancer statistics, 2012. CA Cancer J Clin. 2015;65(2):87-108. doi:10.3322/caac.21262.

5. di Pietro M, Canto MI, Fitzgerald RC. Clinical endoscopic management of early adenocarcinoma and squamous cell carcinoma of the esophagus (screening, diagnosis and therapy). Gastroenterology. Jan. 2018;154(2):421-36. doi:10.1053/j.gastro.2017.07.041.

6. Kufel J, Grzechnik P. Small Nucleolar RNAs Tell a Different Tale. Trends Genet. Dec. 2018. doi:10.1016/j.tig.2018.11.005.

7. 10.1016/j.ygeno.2009.05.002

Dieci G, Preti M, Montanini B, "Eukaryotic snoRNAs: A paradigm for gene expression flexibility," Genomics, vol. 94, no. 2, pp. 83-88, Aug. 2009, doi: 10.1016/j.ygeno.2009.05.002.

8. Siprashvili Z, et al. The noncoding RNAs SNORD50A and SNORD50B bind K-Ras and are recurrently deleted in human cancer. Nat Genet. Jan. 2016;48(1):53-8. doi:10.1038/ng.3452.

9. Matera AG, Terns RM, Terns MP. Non-coding RNAs: lessons from the small nuclear and small nucleolar RNAs. Nat Rev Mol Cell Biol. 2007;8(3):209-20. doi:10.1038/nrm2124.

10. 10.1093/emboj/20.14.3617

Kiss T, "Small nucleolar RNA-guided post-transcriptional modification of cellular RNAs," EMBO J., vol. 20, no. 14, pp. 3617-3622, Jul. 2001, doi: 10.1093/emboj/20.14.3617.

11. Kiss AM, Jády BE, Bertrand E, Kiss T. Human Box H/ACA Pseudouridylation Guide RNA Machinery. Mol Cell Biol. Jul. 2004;24(13):5797-807. doi:10.1128/MCB.24.13.5797-5807.2004.

12. Bellodi C, et al. H/ACA Small RNA Dysfunctions in Disease Reveal Key Roles for Noncoding RNA Modifications in Hematopoietic Stem Cell Differentiation. Cell Reports. May 2013;3(5):1493-502. doi:10.1016/j.celrep.2013.04.030.

13. Williams GT, Farzaneh F. Are snoRNAs and snoRNA host genes new players in cancer? Nat Rev Cancer. Feb. 2012;12(2):84-8. doi:10.1038/nrc3195.

14. Mannoor K, Liao J, Jiang F. "Small nucleolar RNAs in cancer," Biochimica et Biophysica Acta (BBA) Reviews on Cancer, vol. 1826, no. 1, pp. 121-128, Aug. 2012, doi: 10.1016/j.bbcan.2012.03.005.

15. Liao J, et al. Small nucleolar RNA signatures as biomarkers for non-small-cell lung cancer. Molecular Cancer. Jul. 2010;9(1):198. doi:10.1186/1476-4598-9-198.

16. Okugawa $Y$, et al. Clinical significance of SNORA42 as an oncogene and a prognostic biomarker in colorectal cancer. Gut. 2017;66(1):107-17. doi:10.1136/gutjnl-2015-309359. 
17. Mei Y-P, et al. Small nucleolar RNA 42 acts as an oncogene in lung tumorigenesis. Oncogene. May 2012;31(22):2794-804. doi:10.1038/onc.2011.449.

18. Yi C, et al. SNORA42 enhances prostate cancer cell viability, migration and EMT and is correlated with prostate cancer poor prognosis. The International Journal of Biochemistry Cell Biology. Sep. 2018;102:138-50. doi:10.1016/j.biocel.2018.07.009.

19. Abdelhaleem $M$, Maltais $L$, Wain $H$. "The human DDX and DHX gene families of putative RNA helicases," Genomics, vol. 81, no. 6, pp. 618-622, Jun. 2003, doi: 10.1016/S0888-7543(03)00049-1.

20. Abdelhaleem M. "Do human RNA helicases have a role in cancer?," Biochimica et Biophysica Acta (BBA) - Reviews on Cancer, vol. 1704, no. 1, pp. 37-46, Jul. 2004, doi: 10.1016/j.bbcan.2004.05.001.

21. Mi J, et al. In Vivo Selection Against Human Colorectal Cancer Xenografts Identifies an Aptamer That Targets RNA Helicase Protein DHX9. Molecular Therapy - Nucleic Acids. Jan. 2016;5:e315. doi:10.1038/mtna.2016.27.

22. Tetsuka T, et al. RNA helicase A interacts with nuclear factor kappaB p65 and functions as a transcriptional coactivator. Eur J Biochem. Sep. 2004;271(18):3741-51. doi:10.1111/j.14321033.2004.04314.x.

23. 10.1073 /pnas. 1000743107

Huo L, et al., "RNA helicase A is a DNA-binding partner for EGFR-mediated transcriptional activation in the nucleus," Proc. Natl. Acad. Sci. U.S.A., vol. 107, no. 37, pp. 16125-16130, Sep. 2010, doi: 10.1073/pnas.1000743107.

24. Cao S, et al., "RNA helicase DHX9 may be a therapeutic target in lung cancer and inhibited by enoxacin," Am J Transl Res, vol. 9, no. 2, pp. 674-682, Feb. 2017.

25. 10.1038/onc.2016.52

Lee T, Paquet M, Larsson O, Pelletier J, "Tumor cell survival dependence on the DHX9 DExH-box helicase," Oncogene, vol. 35, no. 39, pp. 5093-5105, Sep. 2016, doi: 10.1038/onc.2016.52.

26. Ma M, et al., "p-Hydroxylcinnamaldehyde induces the differentiation of oesophageal carcinoma cells via the cAMP-RhoA-MAPK signalling pathway," Sci Rep, 6, Aug. 2016, doi:10.1038/srep31315.

27. Gong J, et al. A Pan-cancer Analysis of the Expression and Clinical Relevance of Small Nucleolar RNAs in Human Cancer. Cell Reports. Nov. 2017;21(7):1968-81. doi:10.1016/j.celrep.2017.10.070.

28. Tetsuka $T$, et al. RNA helicase $A$ interacts with nuclear factor $K B$ p 65 and functions as a transcriptional coactivator. Eur J Biochem. 2004;271(18):3741-51. doi:10.1111/j.14321033.2004.04314.x.

29. $10.1093 / \mathrm{nar} / \mathrm{gky} 742$

$\mathrm{Ng} \mathrm{YC}$, et al., "A DNA-sensing-independent role of a nuclear RNA helicase, DHX9, in stimulation of NF-KB-mediated innate immunity against DNA virus infection," Nucleic Acids Res, vol. 46, no. 17, pp. 9011-9026, Sep. 2018, doi: 10.1093/nar/gky742.

30. $10.1038 / \mathrm{ncb} 3563$

Zhou F, et al., "AML1-ETO requires enhanced C/D box snoRNA/RNP formation to induce self-renewal Loading [MathJax]/jax/output/CommonHTML/jax.js . 19, no. 7, pp. 844-855, Jul. 2017, doi: 10.1038/ncb3563. 
31. Okugawa $Y$, et al. Clinical significance of SNORA42 as an oncogene and a prognostic biomarker in colorectal cancer. Gut. 2017;66(1):107-17. doi:10.1136/gutjnl-2015-309359.

32. Gao L, et al. Genome-wide small nucleolar RNA expression analysis of lung cancer by nextgeneration deep sequencing. Int J Cancer. 2015;136(6):E623-9. doi:10.1002/ijc.29169.

33. Mei Y-P, et al. Small nucleolar RNA 42 acts as an oncogene in lung tumorigenesis. Oncogene. May 2012;31(22):2794-804. doi:10.1038/onc.2011.449.

34. Yi C, et al. SNORA42 enhances prostate cancer cell viability, migration and EMT and is correlated with prostate cancer poor prognosis. Int J Biochem Cell Biol. Sep. 2018;102:138-50. doi:10.1016/j.biocel.2018.07.009.

35. Zhang S, Grosse F, "Nuclear DNA helicase II unwinds both DNA and RNA," Biochemistry, vol. 33, no. 13, pp. 3906-3912, Apr. 1994.

36. Cao P, et al. Germline Duplication of SNORA18L5 Increases Risk for HBV-related Hepatocellular Carcinoma by Altering Localization of Ribosomal Proteins and Decreasing Levels of p53. Gastroenterology. 2018;155(2):542-56. doi:10.1053/j.gastro.2018.04.020.

37. Christian F, Smith EL, Carmody RJ. "The Regulation of NF-kB Subunits by Phosphorylation," Cells, 5, 1, Mar. 2016, doi:10.3390/cells5010012.

38. $10.1038 /$ ni.2060

Ben-Neriah Y, Karin M, "Inflammation meets cancer, with NF-kB as the matchmaker," Nature Immunology, vol. 12, no. 8, pp. 715-723, Aug. 2011, doi: 10.1038/ni.2060.

39. 10.1158/1078-0432.CCR-09-1352

Arun P, Brown MS, Ehsanian R, Chen Z, Van Waes C, "Nuclear NF-kappaB p65 phosphorylation at serine 276 by protein kinase A contributes to the malignant phenotype of head and neck cancer," Clin. Cancer Res., vol. 15, no. 19, pp. 5974-5984, Oct. 2009, doi: 10.1158/1078-0432.CCR-09-1352.

40. $10.1038 / \mathrm{ncb} 3563$

Zhou F, et al., "AML1-ETO requires enhanced C/D box snoRNA/RNP formation to induce self-renewal and leukaemia," Nature Cell Biology, vol. 19, no. 7, pp. 844-855, Jul. 2017, doi: 10.1038/ncb3563.

41. 10.1038/nature13923

Calo E, Flynn RA, Martin L, Spitale RC, Chang HY, Wysocka J, "RNA helicase DDX21 coordinates transcription and ribosomal RNA processing," Nature, vol. 518, no. 7538, pp. 249-253, Feb. 2015, doi: 10.1038/nature13923.

\section{Figures}



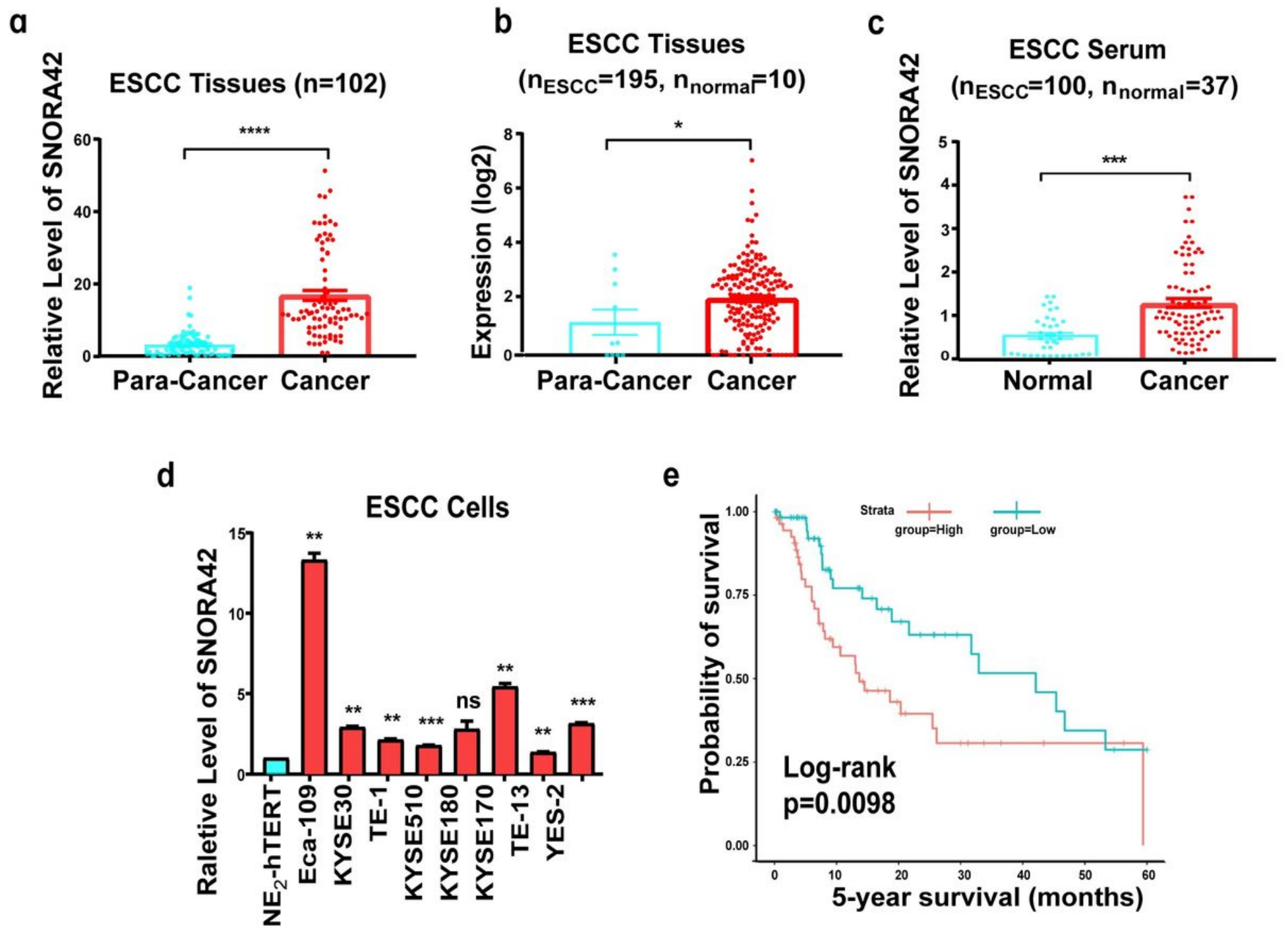

Figure 1

SNORA42 overexpressed in ESCC. (a) The expression of SNORA42 in ESCC tissue and adjacent nontumor tissues from ESCC patients was measured by real-time PCR $(n=102)$. SNORA42 expression in tumor tissues was significantly higher than para-cancer tissues $(* * x p<0.001)$. SNORA42 expression was normalized to that of snRNA U6. (b) The expression level of SNORA42 was overexpressed in esophagus cancer tissues compared with normal esophagus tissues ( $\mathrm{nESCC}=195$, nnormal=10,p=0.0436). Data got fromTCGA database. (c) The expression of SNORA42 in serum from early stages ESCC patients (without radiotherapy, chemotherapy or surgery) and that of healthy volunteers were measured by real-time PCR (nESCC $=100$, nnormal=37). SNORA42 expression in ESCC serum was higher than normal serum (***p<0.001). SNORA42 expression was normalized to that of snRNA U6. (d) The expression of SNORA42 in ESCC cell lines and oesophageal immortalized epithelial cell (NE2-hTERT) was tested by real-time PCR. SNORA42 expression was normalized to that of snRNA U6. (e) The effect of SNORA42 expression on ESCC patents 5-year survival analyzed by Log-rank test (Cox P-value: 4.26e-6, p=0.0098). Data were got from http://bioinfo.life.hust.edu.cn/SNORic/[27]. Data present the mean \pm SEM. Statistical analysis was 
performed using paired two-tailed Student's t-test (a) and unpaired two-tailed Student's t-test (b, c ). ${ }^{* *} p<0.01, * * * p<0.001, * * * * p<0.0001 v s$. controls.

a
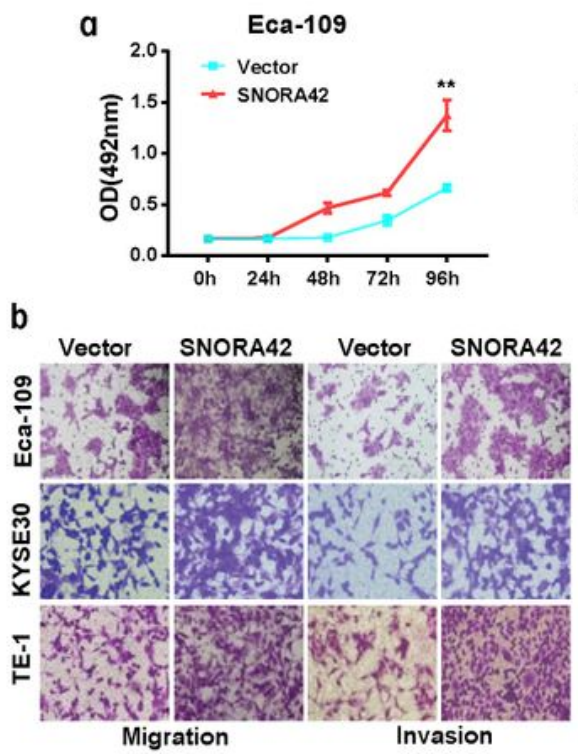

d NC SNORA42- SNORA42-

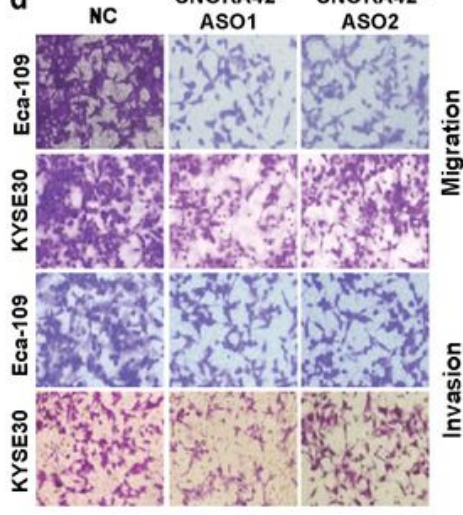

g
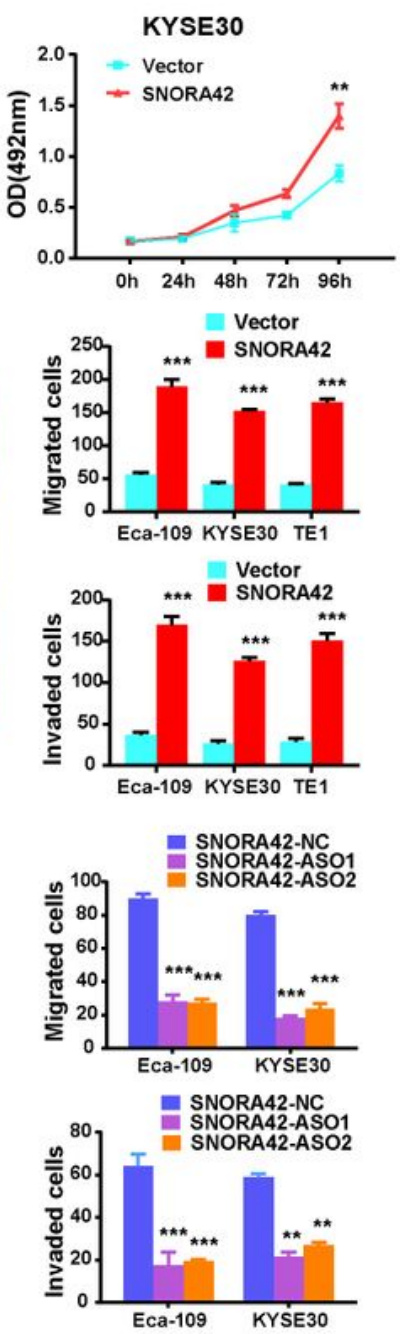

Brain tissue
TE-1
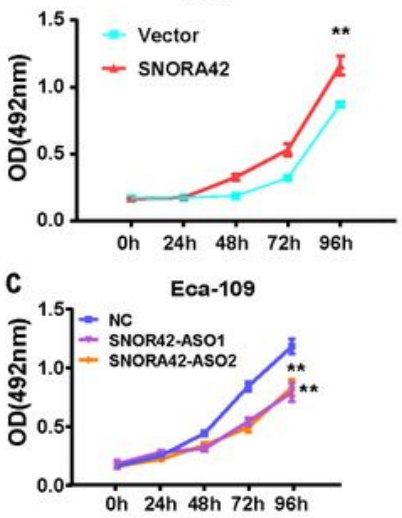

KYSE30

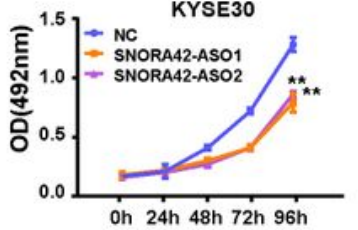

e
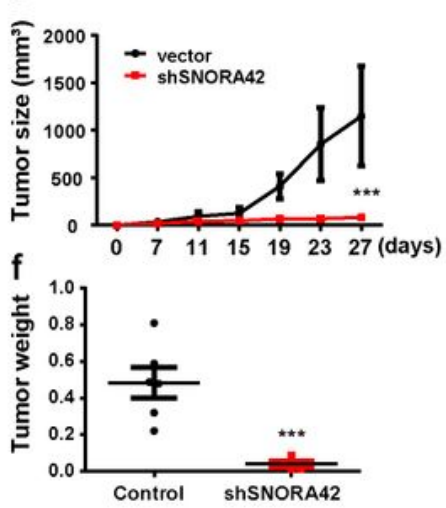

Liver tissue

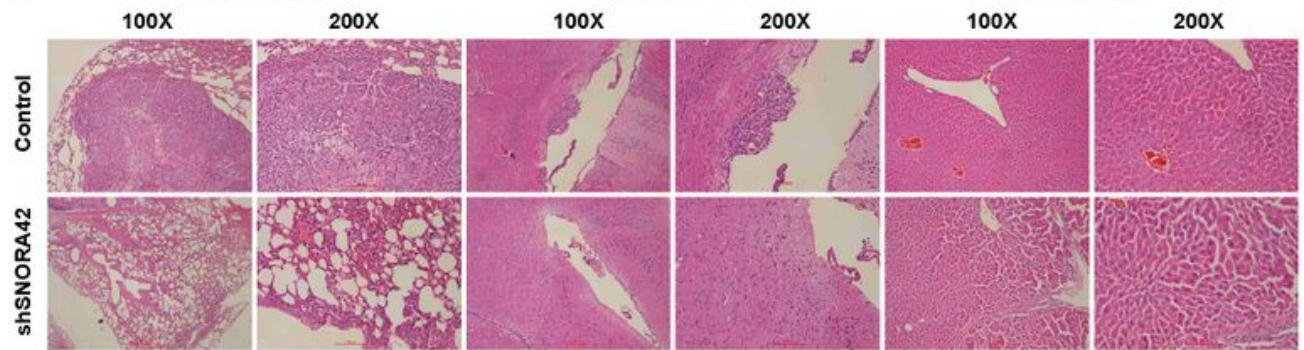

Distant Organ Metastasis

Total Number Control shSNORA42

Lung metastasis

Brain metastasis

Liver metastasis

6
6
6

$\begin{array}{ll}6 & 0 \\ 5 & 1 \\ 0 & 0\end{array}$

$P$-value (Fisher's exact test)

$0.0022^{a}$

0.0801

\section{Figure 2}

SNORA42 exerts tumor-promoting effects in vitro and in vivo. (a) The proliferation of ECSS cells was significantly enhanced by overexpression of SNORA42, assessed by MTS assay. ${ }^{*} p<0.01$. (b) SNORA42 
by Transwell assay and Matrigel assay, independently. ${ }^{\star \star} p<0.01,{ }^{* \star *} p<0.001$. (c) Knockdown of SNORA42 significantly inhibited ESCC cells proliferation. ${ }^{* \star} p<0.01$, ${ }^{\star \star \star} p<0.001$. (d) Knockdown of SNORA42 significantly suppressed ESCC cell migration (upper) and invasion (bottom). ${ }^{\star} p<0.01$, ${ }^{\star \star \star} p<0.001$. (e) The growth of tumor formation with SNORA42 knockdown cell line was slower and smaller than that of negative control cell line in vivo. The two cell lines were subcutaneously injected into armpits of nude mice, independently ( $n=6)$. (f) Knockdown of SNORA42 inhibited the weight of tumor tissues (day 27). (g) The representative H\&E staining images of various organs tissues from the two groups (upper). Knockdown of SNORA42 was significantly inhibited distant organ metastasis of the tumor (bottom). SNORA42 knockdown cell line and negative control cell line were injected through the tail vein $(n=6)$. Data present the mean $\pm \operatorname{SEM}(n=3) .{ }^{* \star} p<0.01,{ }^{* \star *} p<0.001$ vs. controls. 

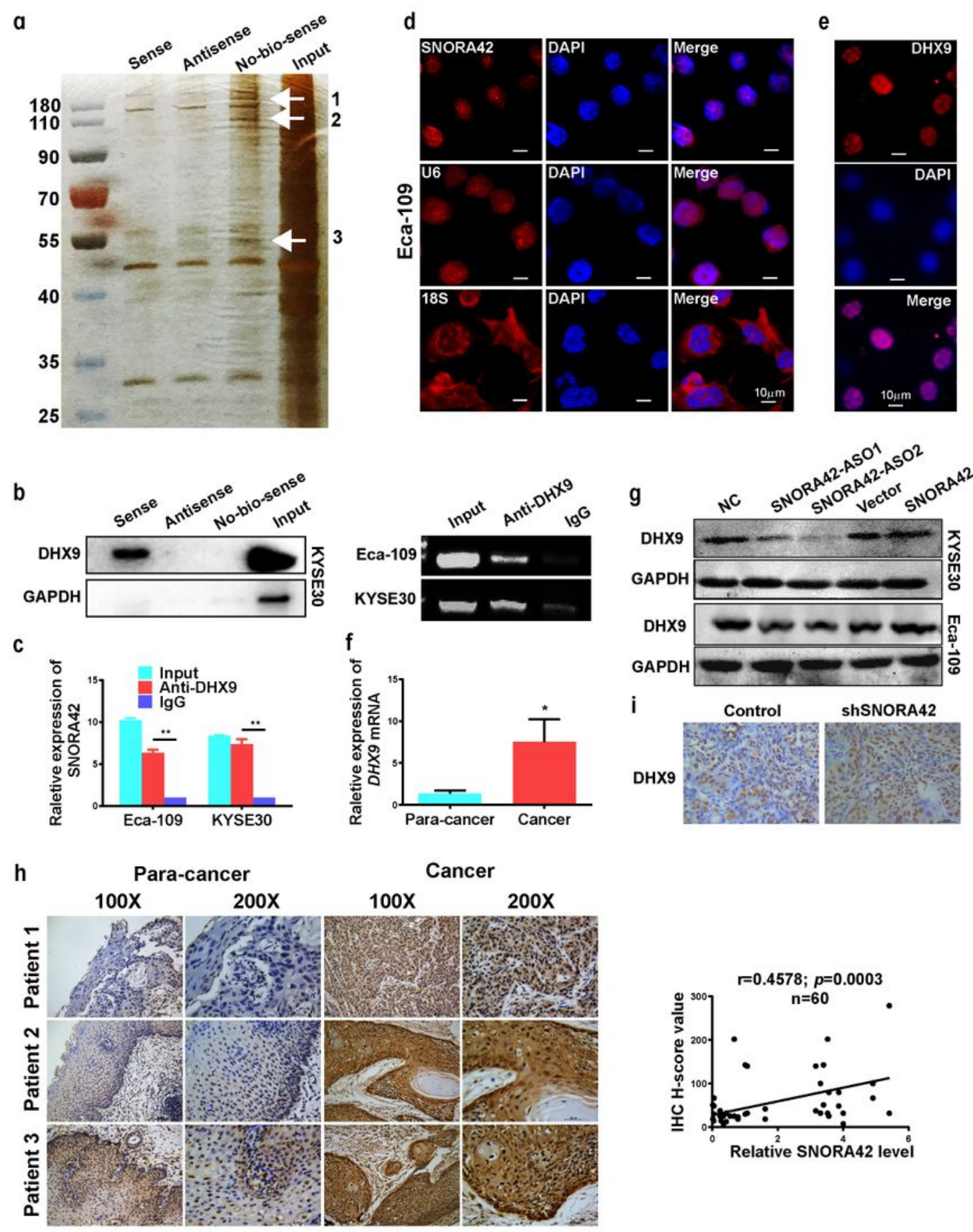

\section{Figure 3}

SNORA42 interacted with DHX9. (a) Identification of the proteins that interacted with SNORA42 was determined by the silver staining. The lysate was extracted from KYSE30 cell and utilized in a biotinylated SNORA42 oligo RNA pull-down assay. The three bands specific associated to SNORA42 were subjected to mass spectrometric analysis. (b) Western Blotting showed the specific interaction of SNORA42 sense 
Immunoprecipitation (RIP) Assay was performed using the indicated antibodies to probe the ESCC cells extracts, and the levels of the co-precipitated RNAs were determined by real-time PCR (bottom) and regular RT-PCR (up). (d) Fluorescence in situ hybridization (FISH) assay was performed using fluorescent labeled SNORA42 probes to localize the cellular location of SNORA42 (upper panel). 18S RNA was used as cytoplasm marker (bottom panel) and snRNA U6 was used as a nuclear marker (middle panel). 4', 6diamidino-2-phenylindole (DAPI) was used to indicate nuclei. (e) Immunofluorescence assay was performed to localize the subcellular location of DHX9. (f) The expression of DHX9gene in ESCC patients was measured by real-time PCR $(n=60)$. DHX9 expression was normalized to GAPDH. (g) DHX9 protein expression is positively associated with knockdown and overexpression of SNORA42 in KYSE30 and Eca109. (h) The representative Immunohistochemical images of DHX9 in ESCC tissues (left), and the correlation of DHX9 protein level and SNORA42 level in the same cohort of ESCC patients (right). Statistical analysis was performed by using Pearson Correlation. (i) Representativelmmunohistochemical images of DHX9 in mice tumor tissues in control and SNORA42knockdown groups. Data present the mean \pm SEM $(n=3) .{ }^{*} p<0.05, * * p<0.01$ vs. controls. 
a
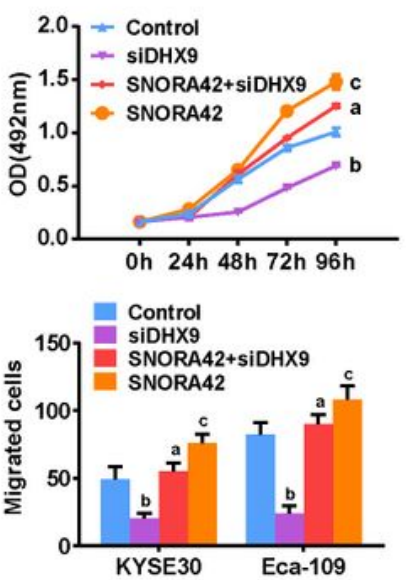
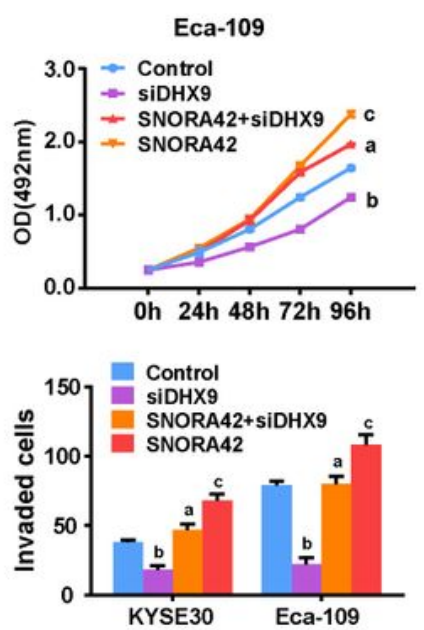

b

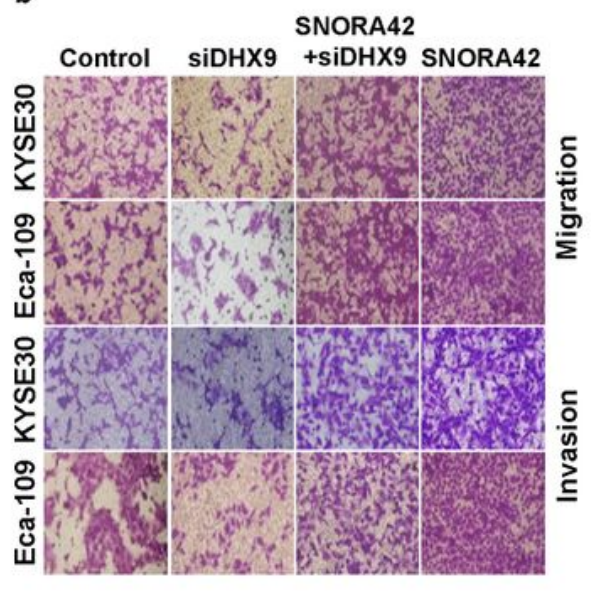

C
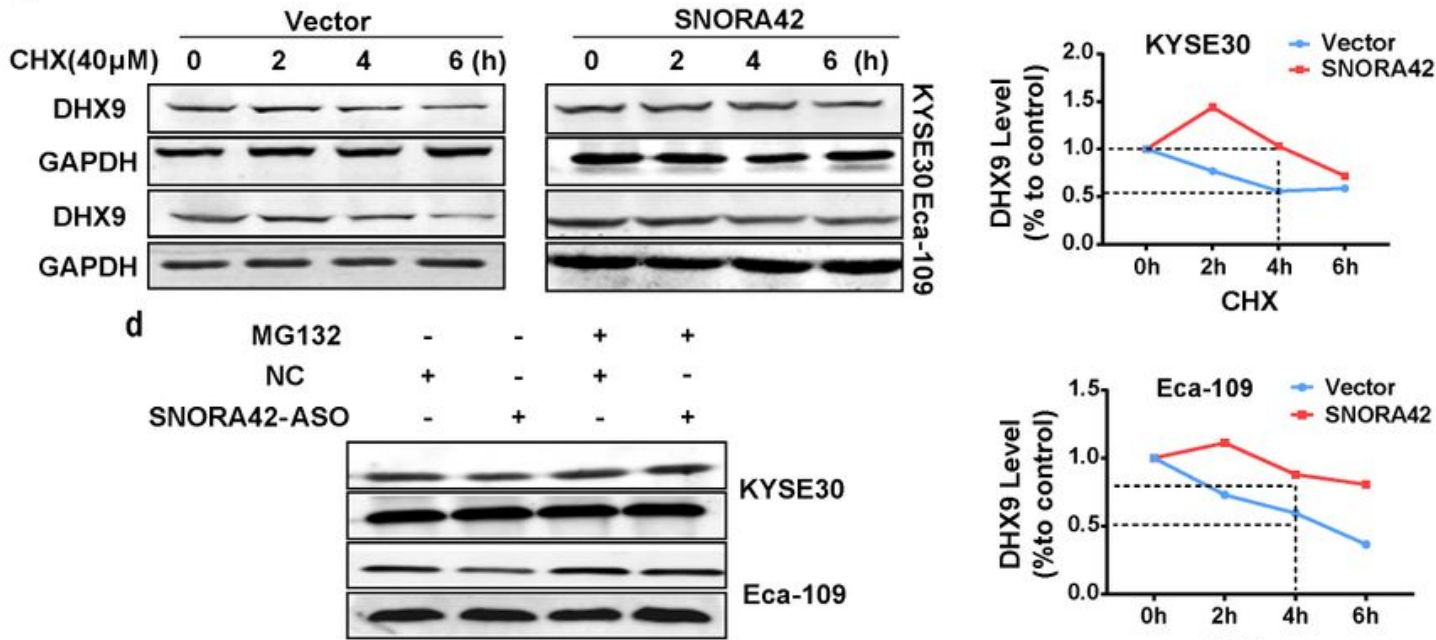

KYSE30

Eca-109

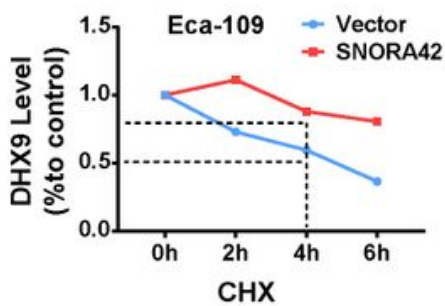

e
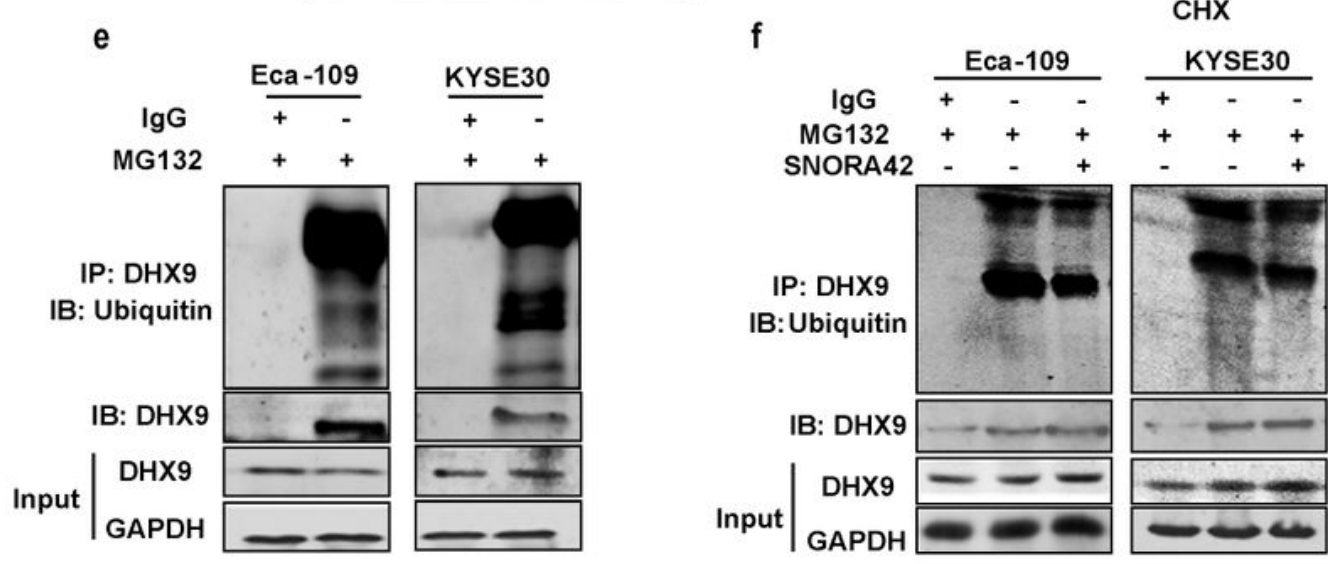

\section{Figure 4}

SNORA42 promotes DHX9 expression by attenuating its ubiquitination.(a) Knocking down of DHX9 reversed the SNORA42-promoted effects on cell proliferation. (b) Knocking down of DHX9 reversed the promoted effects of overexpressed SNORA42 on cell migration (upper panel) and invasion (lower panel). Data present the mean \pm SEM $(n=3)$. Statistical analysis was performed using Student's t-test. Different Loading [MathJax]/jax/output/CommonHTML/jax.js ance. (c) The half-life of DHX9 protein was determined in 


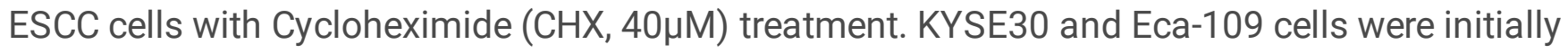
transfected withSNORA42 and vector for 48 hours, and then the cells were treated with CHX. (d) ESCC cells were transfected with NC and SNORA42-ASO for 48 hours, and then treated with MG132 for 6 hours. The immunoblotting assay was performed to measure the expression of DHX9. (e) The lysates of ESCC cells was conducted to immunoprecipitated with the anti-DHX9 antibody or anti-ubiquitination antibody to detect poly-ubiquitination of DHX9 protein. (f) ESCC cells transfected with SNORA42 plasmid or backbone plasmid and the lysates was conducted to immunoprecipitated with same antibodies to detect the level of poly-ubiquitination of DHX9. 


\section{a}

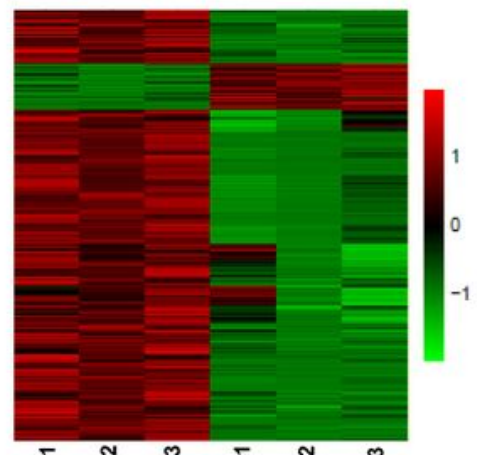

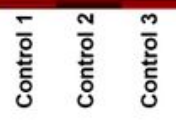

b
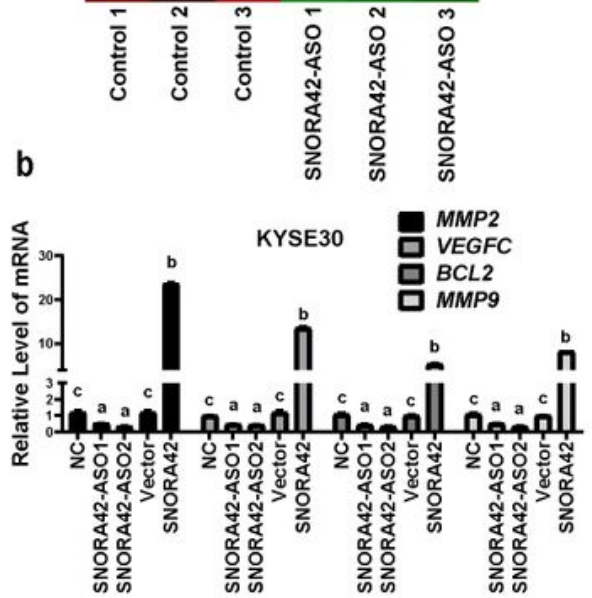

C
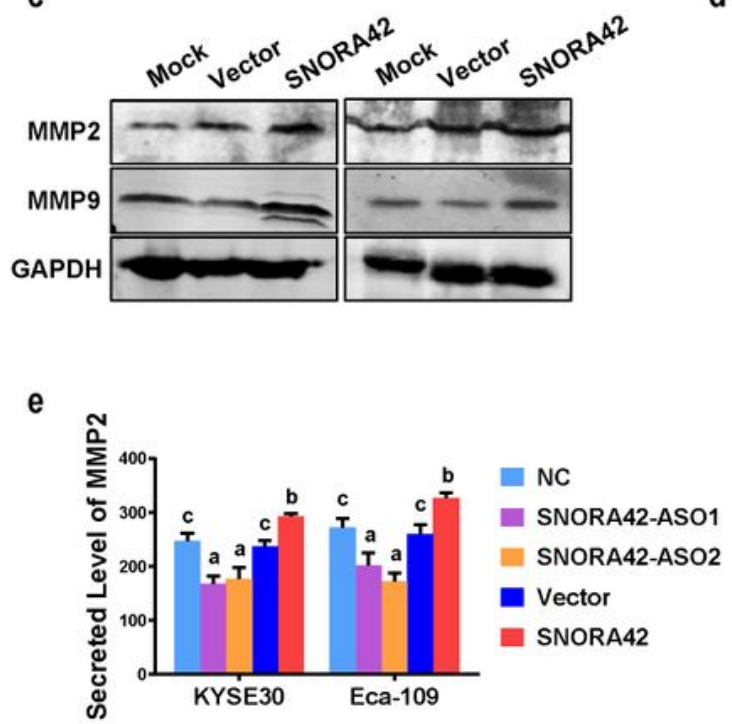

d
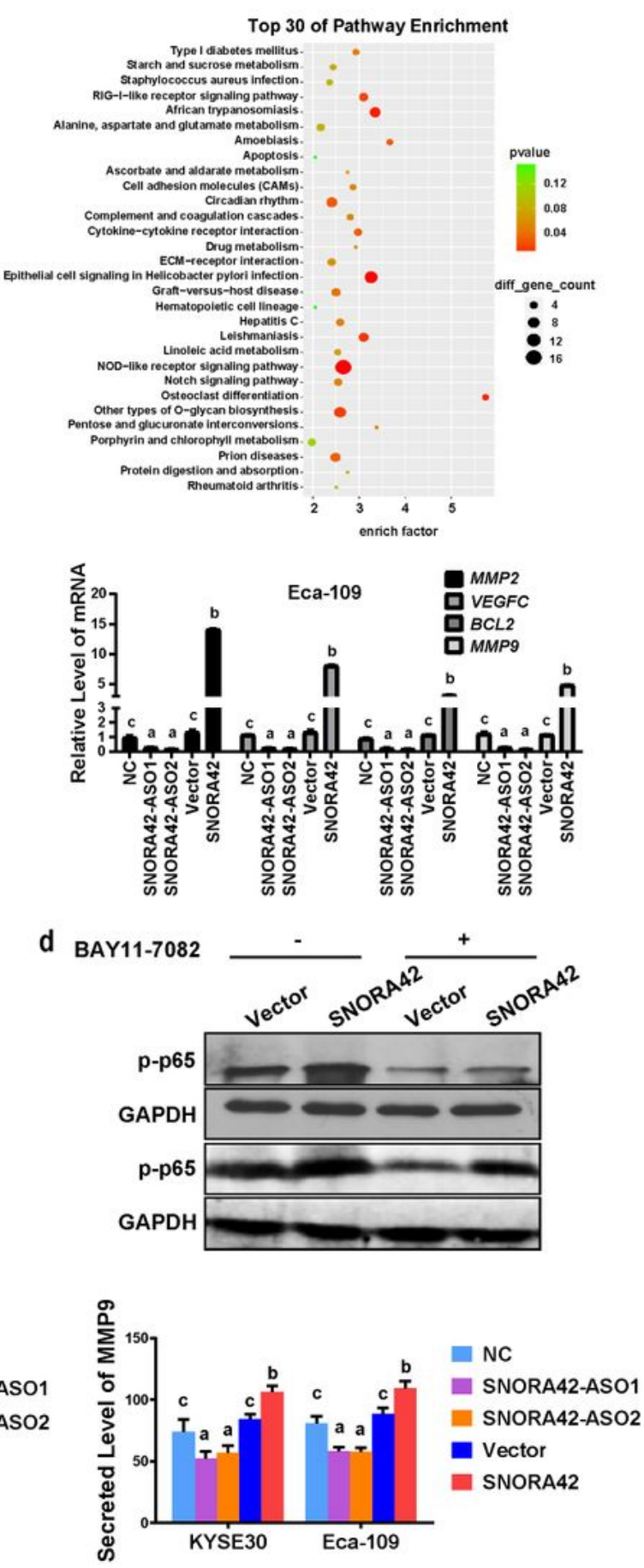

\section{Figure 5}

SNORA42 activates NF-KB pathway. (a) Sequencing analysis of ESCC cells with knocking down of SNORA42 (right panel) and the KEGG pathway analysis from the sequencing results (left panel). (b) Downstream genes of the NF-KB pathway were measured by using real-timePCR. (c) The endogenous levels of MMP2 and MMP9 were tested by Western Blot in SNORA42 overexpressed cells. (d) ELISA was 
SNORA42. (e) p-p65 level was measured in SNORA42 overexpressed cells and treated with NF-kB inhibitor, BAY11-7082. Data present the mean \pm SEM $(n=3)$. Different letters above bars denote statistical significance.

a Eca-109

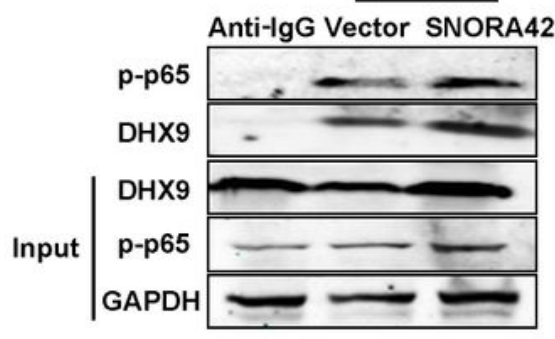

KYSE30

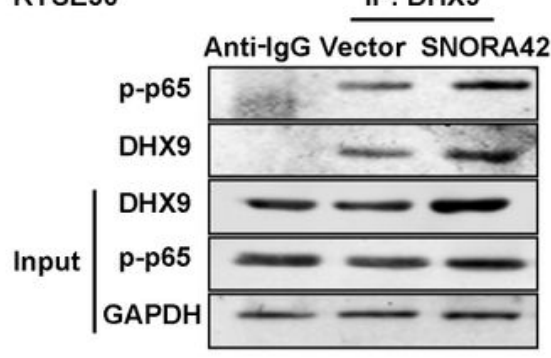

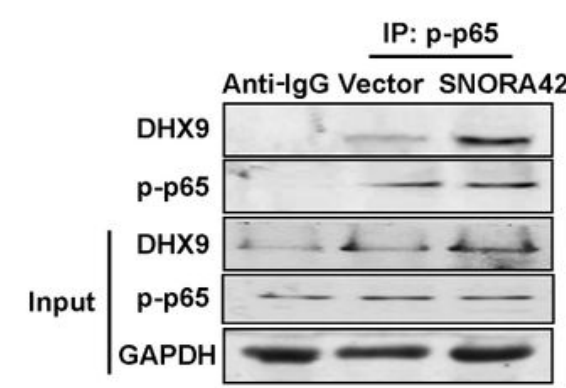

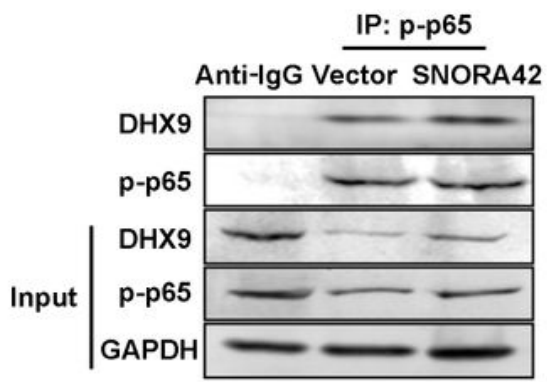

b

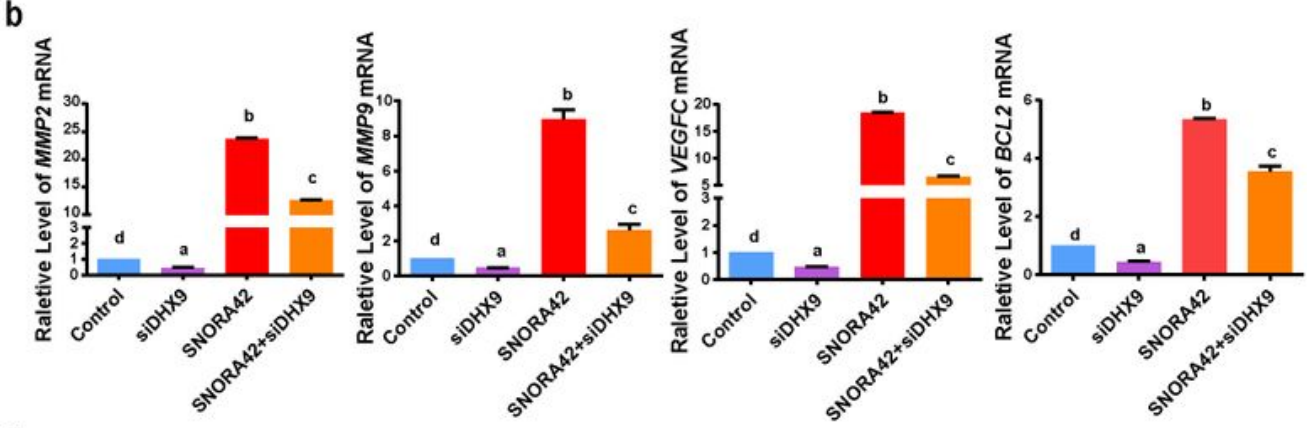

C

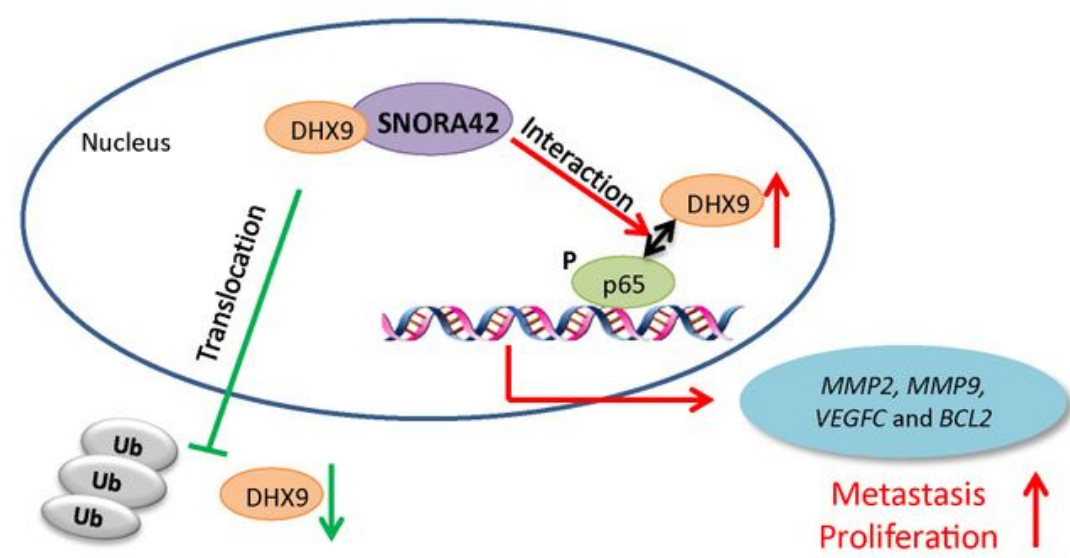

Figure 6

SNORA42 promotes DHX9 interacting with p-p65 to enhance the NF-kB signaling. (a) CoLoading [MathJax]/jax/output/CommonHTML/jax.js ormed using an anti-DHX9 antibody or anti-p-p65 antibody 
on overexpressed SNORA42 ESCC cells. (b) NF-KB downstream genes were tested by real-time PCR after co-transfected siDHX9 and SNORA42 in ESCC cells. Data present the mean \pm SEM $(n=3)$. Different letters above bars denote statistical significance. (c) The schematic of proposed role of SNORA42 in ESCC. SNORA42 is over-expressed in ESCC clinical tissues and cell lines, and promotes ESCC metastasis and proliferation in vitro and in vivo. Mechanically, SNORA42 associates with DHX9 and stabilizes DHX9 protein expression. On the other hand, SNORA42 promotes DHX9 and p-p65 association to further induce p65 downstream genes expression, resulting ESCC metastasis and proliferation.

\section{Supplementary Files}

This is a list of supplementary files associated with this preprint. Click to download.

- Additionaltable2.docx

- Additionaltable2.docx

- Additionaltable2.docx

- Additionaltable1.docx

- Additionaltable1.docx

- Additionaltable1.docx

- Additionaltable3.docx

- Additionaltable3.docx

- Additionaltable3.docx

- additionalfigure3.tiff

- additionalfigure3.tiff

- additionalfigure3.tiff

- additionalfigure2.tiff

- additionalfigure2.tiff

- additionalfigure2.tiff

- additionalfigure1.tiff

- additionalfigure1.tiff

- additionalfigure1.tiff 